\title{
Evaluation of an FFQ to assess total energy and nutrient intakes in severely obese pregnant women
}

\author{
Nor A Mohd-Shukri ${ }^{1}$, Jennifer L Bolton ${ }^{1}$, Jane E Norman ${ }^{2}$, Brian R Walker ${ }^{1}$ and \\ Rebecca M Reynolds, ${ }^{1,2, *}$ \\ 'Endocrinology Unit, University/BHF Centre for Cardiovascular Science, University of Edinburgh, The Queen's \\ Medical Research Institute, 47 Little France Crescent, Edinburgh EH16 4TJ, UK: ${ }^{2}$ Tommy's Centre for Maternal \\ and Fetal Health, MRC Centre for Reproductive Health, University of Edinburgh, The Queen's Medical Research \\ Institute, Edinburgh, UK
}

Submitted 5 December 2011: Final revision received 9 July 2012: Accepted 26 July 2012: First published online 10 September 2012

\begin{abstract}
Objective: FFQ are popular instruments for assessing dietary intakes in epidemiological studies but have not been validated for use in severely obese pregnancy. The aim of the present study was to compare nutrient intakes assessed by an FFQ with those obtained from a food diary among severely obese pregnant women. Design: Comparison of an FFQ containing 170 food items and a food diary for $4 \mathrm{~d}$ (three weekdays and one weekend day); absolute agreement was assessed using the paired $t$ test and relative agreement by Pearson/Spearman correlation, crossclassification into tertiles and weighted kappa values.

Setting: Antenatal metabolic clinic for severely obese women.

Subjects: Thirty-one severely obese (BMI at booking $\geq 40 \cdot 0 \mathrm{~kg} / \mathrm{m}^{2}$ ) and thirty-two lean control $\left(\mathrm{BMI}=20 \cdot 0-24 \cdot 9 \mathrm{~kg} / \mathrm{m}^{2}\right)$ pregnant women.

Results: The findings showed that nutrient intakes estimated by the FFQ were significantly higher than those from the food diary; average correlation was 0.32 in obese and 0.43 in lean women. A mean of $48.5 \%$ of obese and $47.3 \%$ of lean women were correctly classified, while $12.9 \%$ (obese) and $10 \cdot 0 \%$ (lean) were grossly misclassified. Weighted $\kappa$ values ranged from -0.04 to 0.79 in obese women and from $0 \cdot 16$ to $0 \cdot 78$ in lean women.

Conclusions: Overall, the relative agreement between the FFQ and food diary was lower in the obese group than in the lean group, but was comparable with earlier studies conducted in pregnant women. The validity assessments suggest that the FFQ is a useful tool for ranking severely obese pregnant women according to the levels of their dietary intake.
\end{abstract}

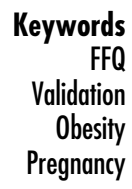

Extra dietary energy is required during pregnancy to compensate for the deposition of maternal and fetal tissues and the increase in $\mathrm{BMR}^{(1)}$. However, with recent data indicating that up to a quarter of adult women are now classified as obese in the $\mathrm{UK}^{(2)}$, including in Scotland $^{(3)}$, the prominent nutritional problem faced by pregnant women in developed countries is overnutrition, including pre-pregnancy overweight/obesity and excessive weight gain during pregnancy. Maternal obesity is associated with detrimental effects in both mothers and offspring. Obese mothers are at higher risk of developing pregnancy complications such as gestational diabetes, pre-eclampsia and having large-for-gestational-age babies ${ }^{(4)}$. Therefore, evaluation of dietary intake in this population is important.

The FFQ is a popular instrument for assessing dietary intakes in epidemiological studies, mostly because it is relatively inexpensive and does not place a heavy burden on either the respondent or research staff. The FFQ also allows collection of information on usual or average food intake over an extended period, thus reflecting habitual intake, rather than a snapshot of a few days' diet ${ }^{(5,6)}$. Therefore FFQ have been used to investigate the nutritional status of pregnant women ${ }^{(7-13)}$, but not severely obese pregnant women.

There are, however, some limitations in the use of the FFQ to estimate food intake. It relies heavily on an individual's ability to recall the foods he/she usually eats and to conceptualize portion sizes. The FFQ is therefore susceptible to measurement errors, as with the use of any dietary assessment instrument ${ }^{(6,14)}$. In addition, because dietary habits differ between populations and FFQ are therefore tailored for specific populations, care must be taken to use an appropriate FFQ to ensure the accuracy of information obtained $^{(5)}$. It is therefore essential to assess the validity of an FFQ in the study population of interest ${ }^{(6)}$. 
There is no consensus in the literature on the best method for validating FFQ. Ideally, FFQ validation should be done against an objective measure of energy expenditure, for example the doubly labelled water technique $^{(15)}$, or by use of other biomarkers such as nitrogen, sodium, vitamin $\mathrm{C}$ and fat-soluble vitamins ${ }^{(16)}$. However, the resources required for these techniques limit their use. Different methods of measuring dietary intake are affected by different sources of bias; however, the validity of one method can be assessed relative to another. A systematic review showed that $75 \%$ of validation studies compared an FFQ against another dietary instrument $^{(6)}$. A food record/diary, with or without food weighing, is commonly used as the reference method for FFQ validation because the food record/diary provides a more detailed and quantitative estimate of nutrient intake, is not influenced by recall bias and is likely to have the smallest correlated errors among dietary assessment instruments $^{(6)}$. A food diary has been used previously to validate an FFQ in pregnant women in the general population $^{(17-19)}$. Various statistical methods are recommended to test the consistency of results from different dietary assessments ${ }^{(6,20)}$.

The Scottish Collaborative Group FFQ (SCG-FFQ) has been used extensively in Scottish pregnant women ${ }^{(9)}$, but its validity and suitability for use in obese pregnant women have never been tested. The present study aimed to compare intakes of total energy and nutrients estimated by the SCG-FFQ with those from a $4 \mathrm{~d}$ food diary (FD; used as the reference method) and to assess the validity and reliability of the SCG-FFQ in the estimating food intake of severely obese pregnant women.

\section{Experimental methods}

\section{Study design and participants}

The study was conducted according to the guidelines laid down in the Declaration of Helsinki and all procedures were approved by the Lothian Research Ethics Committee. Written informed consent was obtained from all participants. Severely obese (BMI at antenatal booking $\geq 40 \cdot 0 \mathrm{~kg} / \mathrm{m}^{2}$ ) and lean (antenatal booking BMI $=20 \cdot 0-24 \cdot 9 \mathrm{~kg} / \mathrm{m}^{2}$ ) pregnant women between 12 and 29 weeks' gestation were recruited from an ongoing cohort study of severe obesity in pregnancy at the Antenatal Metabolic Clinic, Royal Infirmary of Edinburgh, Edinburgh, UK. The women were asked to complete both the SCG-FFQ and FD at the same time point in gestation.

\section{Scottisb Collaborative Group FFQ}

The semi-quantitative SCG-FFQ version 6.6 (Scottish Collaborative Group, University of Aberdeen, Aberdeen, UK) was used, which has been previously validated against $4 \mathrm{~d}$ weighted diet records in non-pregnant individuals $^{(20)}$. The SCG-FFQ consists of a list of 170 foods and drinks which are divided into twenty-one food groups. Women were asked to describe the amount and frequency of consumption of each food on the list over the last 2 to 3 months. This included all meals, snacks and drinks eaten at home or away from home (e.g. at work, at restaurants or cafés and with friends or family). For each food, an example of one measure is provided to help estimate how much of each food is usually eaten (e.g. ' 1 medium slice' for bread). The amount is stated in Measures per Day ( 1 to $5+$ measures) and the frequency in Number of Days per Week ( 1 to $7 \mathrm{~d} /$ week, or ' $\mathrm{R}$ ' for foods which are rarely or never eaten and ' $M$ ' for foods which are consumed between once monthly to less than once weekly). If they chose ' $\mathrm{R}$ ' for frequency, the women were asked to leave the amount section blank. In addition, the SCG-FFQ included a field called 'Other foods and drinks', in which the women could add any other foods or drinks that they usually consumed but were not included in the SCG-FFQ. The SCG-FFQ was checked after completion. If any data were missing, this was discussed with the woman during a clinic visit. Macro- and micronutrient intakes were extracted from questionnaires as previously described ${ }^{(20)}$.

\section{Food diary}

The women were asked to keep a FD (Food and Drink Diary for Adults; University of Aberdeen) for $4 \mathrm{~d}$ (three weekdays and one weekend day), with detailed instructions for data collection. For each day, the women recorded the date, day of the week, time of meal, the type and amount of food and/or drink eaten during each meal and the amount left over, if any. They were asked to record everything they ate and drank at home or outside the home, in as much detail as possible (e.g. by including brand names, the type of bread/dairy products/cereals/ drinks, type of fat or oil used in cooking, methods of cooking and the ingredients used for home-cooked meals). To help them describe the amounts, twenty-two pictures of portion sizes were provided within the food diary, as was an example of one day's record. If they consumed more or less than the amount in the picture, they could describe the amount by writing ' $1 / 2$ ' or ' 2 times', etc. If appropriate, printed weights on packaged foods were used. Women were instructed to record any food left over at the end of the meal to avoid overestimation of food intake. They were advised to record intake at the time of eating, and not from memory at the end of the day. The returned FD was checked for completion. If any information was not complete or not understood, this was addressed with the woman. Data from FD were analysed using the WinDiets Standard dietary analysis software (Robert Gordon University, Aberdeen, UK).

\section{Power calculation}

We aimed for a sample size of between sixteen and thirty-six women in each group based on the numbers 
( $n 36$ men and $n 36$ women) used in the previous validation of the SCG-FFQ in non-pregnant individuals ${ }^{(20)}$ and on the assumptions and methods used in a previous study validating energy intake estimated from an FFQ against the doubly labelled water method ${ }^{(21)}$. The latter study demonstrated that a sample size of sixteen had $80 \%$ power at the 5\% significance level to detect a $16 \%$ difference ( $1400 \mathrm{~kJ})$ between methods.

\section{Statistical analysis}

Normal distribution of data was assessed visually using histograms and Q-Q plots, and by the Shapiro-Wilk test. Total energy and nutrient intakes were not normally distributed and were normalized using natural logarithmic transformation. Significance was reported at $P<0.05$ and all statistical analyses were performed using the SPSS statistical software package version $14 \cdot 0$. Due to non-normal distributions of measured values data are presented as median (25th and 75th percentile), unless stated otherwise.

Within-subject differences between nutrient intakes estimated from the SCG-FFQ and FD were assessed using paired $t$ tests. The relative agreement between the SCG-FFQ and FD was assessed using Spearman rank correlation and Pearson correlation on both reported and energy-adjusted values. The level of agreement between the SCG-FFQ and FD was assessed using Cohen's weighted kappa statistic $\left(\boldsymbol{\kappa}_{\mathrm{w}}\right)$ for each nutrient ${ }^{(22)}$, and the relationship between the methods using cross-classification. Women were classified into tertiles of intake by each dietary method. Percentages of women categorized into the same tertile by both methods or grossly misclassified into opposing tertiles were calculated.

To minimize the effect of measurement errors such as misreporting of energy intake, which would also affect the absolute intakes of nutrients, nutrient intakes were adjusted for total energy intake using the residual method. This was done by adding the residual (the difference between the observed nutrient value for each woman and the value predicted by a regression equation) to the nutrient intake that corresponds with the mean total energy intake of the study population ${ }^{(23)}$. Nutrient intakes from dietary supplements were not included in any of the analyses.

\section{Results}

Forty-eight obese and thirty-seven lean pregnant women agreed to take part, and thirty-one obese (52\%) and thirty-two lean (82\%) pregnant women completed the study. Four FD (from the obese group) were lost in the mail, two lean women withdrew from the study, nine obese and one lean women failed to complete the FD after reminder calls, and four obese and two lean women were not able to be contacted after initially agreeing to take part in the study. The demographic characteristics of women who completed the study are presented in Table 1. Obese and lean groups were of similar age, gestation and parity, and all women were Caucasian. However, obese women had significantly higher levels of socio-economic deprivation (derived from the Deprivation Category Score, which divides postcode sectors into categories in relation to income, employment, health, housing and education) ${ }^{(24)}$ than lean women.

\section{Absolute agreement}

The crude nutrient intakes estimated by the SCG-FFQ and FD are shown in Table 2 (obese group) and Table 3 (lean group). All nutrient intake estimates from the FFQ were significantly higher than those from the FD in both obese and lean groups, with the exception of niacin and vitamin C. Intakes of niacin were estimated to be lower by the SCG-FFQ compared with the FD in both groups. Vitamin C intake was similar between SCG-FFQ and FD in the obese group.

Table 1 Sociodemographic characteristics of the participants: pregnant women between 12 and 29 weeks' gestation, Edinburgh, UK

\begin{tabular}{|c|c|c|c|c|c|}
\hline & \multicolumn{2}{|c|}{ Obese $(n 31)$} & \multicolumn{2}{|c|}{ Lean (n 32) } & \multirow[b]{2}{*}{$P$ value } \\
\hline & Mean or $n$ & Range or \% & Mean or $n$ & Range or \% & \\
\hline Age (years) & $33 \cdot 6$ & $21 \cdot 0-44 \cdot 5$ & $33 \cdot 1$ & $21 \cdot 1-42 \cdot 3$ & $0.672 \dagger$ \\
\hline BMI at booking $\left(\mathrm{kg} / \mathrm{m}^{2}\right)$ & $43 \cdot 3$ & $38 \cdot 6-50 \cdot 2$ & $22 \cdot 8$ & $19 \cdot 9-25 \cdot 4$ & $0.001 t$ \\
\hline \multicolumn{6}{|l|}{ Parity (\%) } \\
\hline Primiparous & 18 & $58 \cdot 1$ & 19 & $59 \cdot 4$ & \multirow[t]{2}{*}{$0.853 \ddagger$} \\
\hline Multiparous & 13 & $41 \cdot 9$ & 13 & $40 \cdot 6$ & \\
\hline \multicolumn{6}{|l|}{ Ethnicity (\%) } \\
\hline Caucasian & 31 & $100 \cdot 0$ & 32 & $100 \cdot 0$ & \\
\hline \multicolumn{6}{|l|}{ DEPCAT status (\%) } \\
\hline Low & 5 & $16 \cdot 1$ & 1 & $9 \cdot 5$ & $0.001 \ddagger$ \\
\hline Middle & 25 & $80 \cdot 6$ & 19 & $69 \cdot 8$ & \\
\hline High & 1 & $3 \cdot 3$ & 12 & $20 \cdot 7$ & \\
\hline Gestation questionnaire completed (weeks) & $26 \cdot 7$ & $12 \cdot 8-28 \cdot 7$ & $27 \cdot 3$ & $12 \cdot 0-29 \cdot 6$ & $0.789+$ \\
\hline
\end{tabular}

DEPCAT, deprivation category ('low' indicates living in the most deprived areas) ${ }^{(23)}$.

†Tested using the independent $t$ test.

$\ddagger$ Tested using the $\chi^{2}$ test. 
Table 2 Median (25th percentile, 75th percentile; $P_{25}, P_{75}$ ) crude daily nutrient intakes estimated from the FFQ and the $4 \mathrm{~d}$ food diary (FD) in the obese group ( $n$ 31): pregnant women between 12 and 29 weeks' gestation, Edinburgh, UK

\begin{tabular}{|c|c|c|c|c|c|c|}
\hline \multirow[b]{2}{*}{ Nutrient } & \multicolumn{2}{|c|}{ FFQ } & \multicolumn{2}{|c|}{ FD } & \multirow[b]{2}{*}{$P$ valuet } & \multirow[b]{2}{*}{$P$ value $\neq$} \\
\hline & Median & $\mathrm{P}_{25}, \mathrm{P}_{75}$ & Median & $\mathrm{P}_{25}, \mathrm{P}_{75}$ & & \\
\hline Energy (kJ) & 10157 & 8453,11262 & 6544 & 5062,7465 & $<0.001$ & - \\
\hline Protein $(g)$ & $103 \cdot 3$ & $78 \cdot 8,124 \cdot 1$ & 63.0 & $50 \cdot 7,72 \cdot 2$ & $<0.001$ & 0.655 \\
\hline Fat $(\mathrm{g})$ & $97 \cdot 9$ & $73 \cdot 2,119 \cdot 8$ & $61 \cdot 6$ & $48 \cdot 4,77 \cdot 0$ & $<0.001$ & 0.827 \\
\hline Carbohydrate (g) & $287 \cdot 8$ & $241 \cdot 6,367 \cdot 0$ & $198 \cdot 7$ & $149 \cdot 2,235 \cdot 4$ & $<0.001$ & $0 \cdot 720$ \\
\hline SFA $(g)$ & $37 \cdot 2$ & $27 \cdot 1,47 \cdot 6$ & $23 \cdot 6$ & $16 \cdot 5,27 \cdot 6$ & $<0.001$ & 0.664 \\
\hline MUFA (g) & $34 \cdot 0$ & $24 \cdot 8,40 \cdot 4$ & $20 \cdot 6$ & $16 \cdot 1,24 \cdot 8$ & $<0.001$ & 0.588 \\
\hline PUFA (g) & $16 \cdot 5$ & $13 \cdot 8,18 \cdot 6$ & $10 \cdot 5$ & $8 \cdot 5,14 \cdot 3$ & 0.004 & $0 \cdot 168$ \\
\hline Cholesterol (mg) & 300 & 215,420 & 185 & 133,222 & $<0.001$ & 0.466 \\
\hline Sugars (g) & $134 \cdot 0$ & $113 \cdot 8,167 \cdot 0$ & $80 \cdot 3$ & $62 \cdot 0,101 \cdot 4$ & $<0.001$ & $0 \cdot 105$ \\
\hline Starch $(\mathrm{g})$ & $138 \cdot 0$ & $121 \cdot 0,178 \cdot 0$ & $112 \cdot 1$ & $97 \cdot 0,132 \cdot 9$ & 0.001 & 0.362 \\
\hline Fibre $(\mathrm{g})$ & $22 \cdot 0$ & $16 \cdot 1,28 \cdot 3$ & $3 \cdot 3$ & $2 \cdot 7,4 \cdot 3$ & $<0.001$ & $1 \cdot 000$ \\
\hline $\mathrm{Na}(\mathrm{mg})$ & 3222 & 2448,3972 & 2381 & 1884, 2782 & 0.002 & 0.293 \\
\hline $\mathrm{K}(\mathrm{mg})$ & 3957 & 3300,5166 & 2371 & 1860,2834 & $<0.001$ & 0.686 \\
\hline $\mathrm{Ca}(\mathrm{mg})$ & 1197 & 922,1596 & 776 & 584,945 & $<0.001$ & 0.735 \\
\hline $\mathrm{Mg}(\mathrm{mg})$ & 416 & 338,490 & 211 & 152,268 & $<0.001$ & 0.279 \\
\hline $\mathrm{P}(\mathrm{mg})$ & 1783 & 1448,2178 & 1075 & 890,1230 & $<0.001$ & 0.558 \\
\hline $\mathrm{Fe}(\mathrm{mg})$ & $14 \cdot 17$ & $11 \cdot 54,17 \cdot 34$ & $8 \cdot 40$ & $6 \cdot 05,11 \cdot 25$ & $<0.001$ & 0.504 \\
\hline $\mathrm{Cu}(\mathrm{mg})$ & $2 \cdot 83$ & $2 \cdot 36,3 \cdot 47$ & $0 \cdot 81$ & $0.53,1 \cdot 11$ & $<0.001$ & 0.854 \\
\hline $\mathrm{Zn}(\mathrm{mg})$ & $12 \cdot 40$ & $9 \cdot 50,15 \cdot 40$ & $6 \cdot 60$ & $5 \cdot 65,8 \cdot 30$ & $<0.001$ & 0.671 \\
\hline $\mathrm{Cl}(\mathrm{mg})$ & 4826 & 3788,6061 & 3575 & 2772,4036 & 0.002 & $0 \cdot 165$ \\
\hline $\mathrm{Mn}(\mathrm{mg})$ & $4 \cdot 30$ & $3 \cdot 30,4 \cdot 95$ & $2 \cdot 08$ & $1 \cdot 51,2 \cdot 71$ & $<0.001$ & 0.583 \\
\hline $\operatorname{Se}(\mu \mathrm{g})$ & $67 \cdot 0$ & $47 \cdot 0,80 \cdot 0$ & $34 \cdot 0$ & $27 \cdot 5,47 \cdot 5$ & $<0.001$ & 0.570 \\
\hline lodine $(\mu \mathrm{g})$ & 254 & 182,316 & 122 & 96,152 & $<0.001$ & 0.692 \\
\hline Retinol $(\mu \mathrm{g})$ & 325 & 236,426 & 224 & 171,326 & 0.012 & 0.690 \\
\hline$\beta$-Carotene equivalents $(\mu \mathrm{g})$ & 3381 & 2393,7336 & 1093 & 826,1656 & $<0.001$ & 0.800 \\
\hline Vitamin D $(\mu \mathrm{g})$ & 4.05 & $2 \cdot 61,4 \cdot 77$ & $1 \cdot 79$ & $0.72,2 \cdot 54$ & $<0.001$ & 0.820 \\
\hline Vitamin E (mg) & 11.98 & $9 \cdot 03,15 \cdot 33$ & $7 \cdot 11$ & $5.07,9.03$ & $<0.001$ & 0.800 \\
\hline Thiamin (mg) & 1.97 & $1 \cdot 53,2 \cdot 40$ & $1 \cdot 36$ & $1 \cdot 11,1 \cdot 59$ & $<0.001$ & $0 \cdot 145$ \\
\hline Riboflavin (mg) & $2 \cdot 08$ & $1 \cdot 60,2 \cdot 89$ & $1 \cdot 30$ & $1 \cdot 01,1.61$ & $<0.001$ & 0.970 \\
\hline Niacin (mg) & $24 \cdot 50$ & $18 \cdot 55,29 \cdot 85$ & $27 \cdot 80$ & $23 \cdot 65,34 \cdot 80$ & 0.011 & 0.509 \\
\hline Vitamin $B_{6}(\mathrm{mg})$ & $2 \cdot 58$ & $2 \cdot 03,3 \cdot 30$ & 1.59 & $1 \cdot 40,2 \cdot 12$ & $<0.001$ & $0 \cdot 155$ \\
\hline Vitamin $B_{12}(\mu \mathrm{g})$ & $6 \cdot 80$ & $5 \cdot 05,8 \cdot 80$ & 3.89 & $2 \cdot 78,5 \cdot 43$ & $<0.001$ & 0.541 \\
\hline Folic acid $(\mu \mathrm{g})$ & 323 & 240,398 & 204 & 154,231 & $<0.001$ & 0.390 \\
\hline Pantothenic acid (mg) & 6.53 & $5 \cdot 20,7 \cdot 91$ & 3.60 & $3 \cdot 15,5 \cdot 00$ & $<0.001$ & 0.807 \\
\hline Biotin $(\mu \mathrm{g})$ & $46 \cdot 5$ & $36 \cdot 7,52 \cdot 4$ & $20 \cdot 3$ & $16 \cdot 4,26 \cdot 5$ & $<0.001$ & 0.916 \\
\hline Vitamin C (mg) & $150 \cdot 0$ & $116 \cdot 5,229 \cdot 5$ & $99 \cdot 4$ & $49 \cdot 5,173 \cdot 2$ & 0.197 & 0.907 \\
\hline
\end{tabular}

t $P$ value, log-transformed as necessary, crude intakes (paired $t$ test).

$\ddagger P$ value, log-transformed as necessary, energy-adjusted intakes (paired $t$ test).

\section{Relative agreement}

Tables 4 and 5 show the Pearson and Spearman correlation coefficients between nutrient intakes estimated by the SCG-FFQ and FD, for obese and lean groups respectively. For energy-adjusted data, Pearson correlation coefficients were similar in obese (range $-0 \cdot 19$ to $0 \cdot 71$ ) and lean (range $-0 \cdot 25$ to $0 \cdot 74$ ) groups. There was a significant correlation for total energy intake between the SCG-FFQ and FD in the lean group, but not in the obese group. In terms of macronutrients, in obese women there was a significant correlation between the two methods for fat, which did not persist when adjusted for total energy; whereas the correlation for protein became significant after energy adjustment. In lean women, there was a significant correlation for protein, but this was no longer significant after adjustment for total energy.

A correlation coefficient between dietary assessment methods of greater than 0.5 has been suggested as acceptable in validation studies of $\mathrm{FFQ}^{(25)}$. In the present study, Pearson correlation coefficients $>0.5$ were found for measurements of one nutrient (dietary cholesterol) in obese women and fifteen nutrients in lean women. After energy adjustment, Pearson correlation coefficients $>0.5$ were found for five nutrients in obese and eight nutrients in lean women. In obese women, significant correlation coefficients were observed for nine nutrients including SFA, dietary cholesterol, sugars, Ca, $\mathrm{P}$, iodine and vitamins $\mathrm{B}_{6}, \mathrm{~B}_{12}$ and $\mathrm{C}$, both before and after adjustment for total energy. In lean women, this was observed for a total of eighteen nutrients (SFA, PUFA, dietary cholesterol, fibre, $\mathrm{K}$, Ca, $\mathrm{P}, \mathrm{Zn}, \mathrm{Mn}$, iodine, $\beta$-carotene, riboflavin, niacin, biotin and vitamins $\mathrm{B}_{6}, \mathrm{~B}_{9}$ and $\mathrm{C}$ ). Correlation coefficients were lowest for fibre and Se in obese women, and carbohydrate and $\mathrm{Cu}$ in lean women.

The ability of the FFQ to rank the participants was determined by cross-classification. Percentages of women categorized into the same tertile or opposite tertiles of intake by each method, and associated $\kappa_{\mathrm{w}}$ statistics for each nutrient, are shown in Table 6. On average, about $48 \%$ of women in both groups were correctly 
Table 3 Median (25th percentile, 75th percentile; $P_{25}, P_{75}$ ) crude daily nutrient intakes estimated from the FFQ and the $4 d$ food diary (FD) in the lean group ( $n 32)$ : pregnant women between 12 and 29 weeks' gestation, Edinburgh, UK

\begin{tabular}{|c|c|c|c|c|c|c|}
\hline \multirow[b]{2}{*}{ Nutrient } & \multicolumn{2}{|c|}{ FFQ } & \multicolumn{2}{|c|}{ FD } & \multirow[b]{2}{*}{$P$ valuet } & \multirow[b]{2}{*}{$P$ value $\ddagger$} \\
\hline & Median & $\mathrm{P}_{25}, \mathrm{P}_{75}$ & Median & $\mathrm{P}_{25}, \mathrm{P}_{75}$ & & \\
\hline Energy (kJ) & 10421 & 8415,12644 & 7335 & 6167,8131 & $<0.001$ & - \\
\hline Protein (g) & $92 \cdot 6$ & $74 \cdot 2,122 \cdot 0$ & $65 \cdot 8$ & $56 \cdot 2,74 \cdot 6$ & $<0.001$ & 0.782 \\
\hline Fat (g) & $95 \cdot 4$ & $72 \cdot 4,129 \cdot 0$ & $66 \cdot 1$ & $54 \cdot 9,81 \cdot 7$ & $<0.001$ & 0.873 \\
\hline Carbohydrate (g) & $334 \cdot 9$ & $259 \cdot 4,358 \cdot 4$ & $222 \cdot 8$ & $188 \cdot 6,263 \cdot 0$ & $<0.001$ & $0 \cdot 819$ \\
\hline SFA (g) & $36 \cdot 2$ & $27 \cdot 4,50 \cdot 4$ & $22 \cdot 5$ & $19 \cdot 8,28 \cdot 9$ & $<0.001$ & 0.651 \\
\hline MUFA (g) & $33 \cdot 1$ & $25 \cdot 0,43 \cdot 3$ & $21 \cdot 8$ & $17 \cdot 6,25 \cdot 0$ & $<0.001$ & 0.528 \\
\hline PUFA (g) & $17 \cdot 5$ & $13 \cdot 0,21 \cdot 0$ & $12 \cdot 0$ & $9 \cdot 3,13 \cdot 9$ & $<0.001$ & 0.072 \\
\hline Cholesterol (mg) & 321 & 205,388 & 210 & 144,253 & $<0.001$ & $0 \cdot 364$ \\
\hline Sugars (g) & $144 \cdot 1$ & $114 \cdot 1,188 \cdot 2$ & $107 \cdot 5$ & $80 \cdot 4,123 \cdot 4$ & $<0.001$ & $0 \cdot 116$ \\
\hline Starch $(g)$ & $170 \cdot 5$ & $130 \cdot 2,194 \cdot 2$ & $117 \cdot 8$ & $96 \cdot 8,140 \cdot 9$ & $<0.001$ & 0.529 \\
\hline Fibre $(\mathrm{g})$ & $24 \cdot 8$ & $19 \cdot 4,30 \cdot 0$ & $5 \cdot 1$ & $3 \cdot 0,6 \cdot 5$ & $<0.001$ & 0.952 \\
\hline $\mathrm{Na}(\mathrm{mg})$ & 3299 & 2440,4433 & 2336 & 2005, 2748 & $<0.001$ & 0.302 \\
\hline $\mathrm{K}(\mathrm{mg})$ & 4294 & 3420,4928 & 2371 & 2104, 2994 & $<0.001$ & $0 \cdot 844$ \\
\hline $\mathrm{Ca}(\mathrm{mg})$ & 1372 & 1035,1609 & 831 & 788,1110 & $<0.001$ & 0.715 \\
\hline $\mathrm{Mg}(\mathrm{mg})$ & 468 & 389,539 & 239 & 207, 301 & $<0.001$ & 0.417 \\
\hline $\mathrm{P}(\mathrm{mg})$ & 1931 & 1485,2343 & 1169 & 1046,1312 & $<0.001$ & 0.481 \\
\hline $\mathrm{Fe}(\mathrm{mg})$ & $17 \cdot 68$ & $13 \cdot 70,21 \cdot 34$ & $10 \cdot 45$ & $8 \cdot 55,12 \cdot 13$ & $<0.001$ & 0.630 \\
\hline $\mathrm{Cu}(\mathrm{mg})$ & $3 \cdot 27$ & $2 \cdot 76,4 \cdot 36$ & $1 \cdot 06$ & $0 \cdot 86,1 \cdot 25$ & $<0.001$ & $0 \cdot 856$ \\
\hline Zn (mg) & $12 \cdot 55$ & $8 \cdot 78,15 \cdot 25$ & $7 \cdot 30$ & $6 \cdot 28,8 \cdot 90$ & $<0.001$ & 0.678 \\
\hline $\mathrm{Cl}(\mathrm{mg})$ & 4986 & 3667,6549 & 3362 & 2877,3810 & $<0.001$ & $0 \cdot 161$ \\
\hline $\mathrm{Mn}(\mathrm{mg})$ & $4 \cdot 40$ & $3 \cdot 98,6 \cdot 33$ & $2 \cdot 66$ & $2 \cdot 26,3 \cdot 32$ & $<0.001$ & 0.625 \\
\hline Se $(\mu \mathrm{g})$ & $67 \cdot 0$ & $50 \cdot 0,82 \cdot 0$ & $36 \cdot 5$ & $26 \cdot 8,48 \cdot 2$ & $<0.001$ & 0.490 \\
\hline lodine $(\mu \mathrm{g})$ & 276 & 193,368 & 152 & 112,174 & $<0.001$ & 0.746 \\
\hline Retinol $(\mu \mathrm{g})$ & 339 & 252,506 & 297 & 209,345 & 0.028 & 0.592 \\
\hline$\beta$-Carotene equivalents $(\mu \mathrm{g})$ & 3925 & 2890,8520 & 1608 & 951,2088 & $<0.001$ & 0.699 \\
\hline Vitamin D $(\mu \mathrm{g})$ & $4 \cdot 68$ & $2 \cdot 88,6 \cdot 53$ & $2 \cdot 24$ & $1 \cdot 17,3 \cdot 53$ & $<0.001$ & 0.781 \\
\hline Vitamin E (mg) & $13 \cdot 69$ & $9 \cdot 38,15 \cdot 76$ & $8 \cdot 51$ & $6 \cdot 63,10 \cdot 71$ & $<0.001$ & $0 \cdot 740$ \\
\hline Thiamin (mg) & $2 \cdot 19$ & $1 \cdot 72,2 \cdot 48$ & $1 \cdot 43$ & $1 \cdot 19,1 \cdot 55$ & $<0.001$ & 0.242 \\
\hline Riboflavin (mg) & $2 \cdot 46$ & $2 \cdot 03,2 \cdot 87$ & 1.66 & $1 \cdot 41,1 \cdot 82$ & $<0.001$ & 0.970 \\
\hline Niacin (mg) & $24 \cdot 55$ & $19 \cdot 73,28 \cdot 15$ & $29 \cdot 90$ & $26 \cdot 05,34 \cdot 98$ & $<0.001$ & 0.405 \\
\hline Vitamin $B_{6}(\mathrm{mg})$ & $2 \cdot 84$ & $2 \cdot 11,3 \cdot 37$ & $1 \cdot 53$ & $1 \cdot 32,1 \cdot 81$ & $<0.001$ & $0 \cdot 193$ \\
\hline Vitamin $B_{12}(\mu \mathrm{g})$ & $7 \cdot 35$ & $4 \cdot 80,9 \cdot 83$ & $3 \cdot 65$ & $2 \cdot 71,5 \cdot 04$ & $<0.001$ & 0.594 \\
\hline Folic acid $(\mu \mathrm{g})$ & 398 & 285,450 & 230 & 182,262 & $<0.001$ & 0.587 \\
\hline Pantothenic acid (mg) & $7 \cdot 07$ & $5 \cdot 27,8 \cdot 46$ & $4 \cdot 20$ & $3 \cdot 58,5 \cdot 13$ & $<0.001$ & $0 \cdot 840$ \\
\hline Biotin $(\mu \mathrm{g})$ & $50 \cdot 0$ & $36 \cdot 5,61 \cdot 4$ & $26 \cdot 1$ & $22 \cdot 2,33 \cdot 7$ & $<0.001$ & 0.962 \\
\hline Vitamin C (mg) & $154 \cdot 5$ & $116 \cdot 0,221 \cdot 2$ & $108 \cdot 5$ & $77 \cdot 6,149 \cdot 5$ & 0.001 & 0.970 \\
\hline
\end{tabular}

† $P$ value, log-transformed as necessary, crude intakes (paired $t$ test).

$\ddagger P$ value, log-transformed as necessary, energy-adjusted intakes (paired $t$ test).

classified, whereas $13 \%$ of obese and $10 \%$ of lean women were grossly misclassified between the two methods. The $\kappa_{\mathrm{w}}$ value for total energy was lower in the obese group compared with the lean group. In general, $\boldsymbol{\kappa}_{\mathrm{w}}<0$ indicates poor agreement, $\boldsymbol{\kappa}_{\mathrm{W}}=0-0 \cdot 2$ indicates slight agreement, $\boldsymbol{\kappa}_{\mathrm{W}}=0 \cdot 2-0 \cdot 4$ fair agreement, $\boldsymbol{\kappa}_{\mathrm{w}}=0 \cdot 4-0 \cdot 6$ moderate agreement and $\boldsymbol{\kappa}_{\mathrm{w}}=0 \cdot 6-0 \cdot 8$ indicates substantial agreement ${ }^{(26)}$. Within macronutrients, substantial agreement was observed for fat in obese and protein in lean groups; moderate agreement was observed for protein in obese and fat in lean groups; and fair agreement for carbohydrates in both groups. There was poor agreement for fibre in the obese (in agreement with cross-classification and correlation) group and slight agreement for $\mathrm{Cu}$, Se and vitamin $\mathrm{B}_{12}$ in the lean group. However, substantial agreement was shown for fat, SFA, PUFA, $\mathrm{Cl}$, iodine and retinol in obese women, and for protein, SFA, PUFA, cholesterol, $\mathrm{Ca}, \mathrm{P}, \mathrm{Fe}, \mathrm{Zn}, \mathrm{Mn}$, iodine, retinol, niacin and vitamin $\mathrm{B}_{6}$ in lean women.

\section{Discussion}

In the present study of pregnant Scottish women aged 21 to 42 years, nutrient intakes estimated by the SCG-FFQ were compared with those recorded in a $4 \mathrm{~d}$ unweighed FD to evaluate the validity of the SCG-FFQ in severely obese pregnant women $\left(\mathrm{BMI} \geq 40 \cdot 0 \mathrm{~kg} / \mathrm{m}^{2}\right)$. The principal use of the FFQ is to categorize individuals according to relative nutrient intakes, i.e. to distinguish people with low intakes from those with high intakes, to avoid the necessity of assessing absolute intakes of nutrients ${ }^{(27)}$. An FFQ's validity can be evaluated by comparing it with other dietary assessment tools such as food records, or with biochemical measurements of energy or nutrients ${ }^{(6)}$. A $4 \mathrm{~d}$ FD was chosen as the reference method in the present study due to its feasibility, detailed and quantitative estimates, and non-reliance on the participant's memory or ability to estimate portion sizes.

Assessments of absolute nutrient intakes between the two methods found that the SCG-FFQ generally reported 
Table 4 Pearson $\left(r_{\mathrm{P}}\right)$ and Spearman $\left(r_{\mathrm{S}}\right)$ correlation coefficients between the FFQ and $4 \mathrm{~d}$ food diary in the obese group ( $n 31$ ): pregnant women between 12 and 29 weeks' gestation, Edinburgh, UK

\begin{tabular}{|c|c|c|c|c|}
\hline \multirow[b]{2}{*}{ Nutrient } & \multicolumn{2}{|c|}{ Pearsont } & \multicolumn{2}{|c|}{ Spearman } \\
\hline & $r_{\mathrm{P}}^{\text {measured }}$ & $r_{\mathrm{P}}^{\text {energy-adjusted }}$ & $r_{\mathrm{S}}^{\text {measured }}$ & $r_{\mathrm{S}}^{\text {energy-adjusted }}$ \\
\hline Energy (kJ) & 0.28 & - & 0.33 & - \\
\hline Protein $(\mathrm{g})$ & $0 \cdot 19$ & $0.35^{*}$ & 0.25 & $0 \cdot 30$ \\
\hline Fat $(\mathrm{g})$ & $0 \cdot 38^{*}$ & $0 \cdot 12$ & $0 \cdot 49^{\star *}$ & -0.01 \\
\hline Carbohydrate (g) & 0.23 & -0.03 & $0 \cdot 26$ & 0.20 \\
\hline SFA (g) & $0.50^{\star *}$ & $0 \cdot 45^{\star \star}$ & $0.52^{\star \star}$ & $0 \cdot 30$ \\
\hline MUFA (g) & $0 \cdot 43^{*}$ & $0 \cdot 18$ & $0.52^{\star *}$ & 0.09 \\
\hline PUFA (g) & $0 \cdot 19$ & $0 \cdot 16$ & 0.20 & $0 \cdot 15$ \\
\hline Cholesterol (mg) & $0.54^{\star \star}$ & $0 \cdot 46^{\star \star}$ & $0.42^{*}$ & 0.22 \\
\hline Sugars (g) & $0 \cdot 35^{\star}$ & $0.58^{\star *}$ & $0.44^{*}$ & 0.25 \\
\hline Starch (g) & 0.29 & $0 \cdot 42^{*}$ & $0.39^{*}$ & $0 \cdot 21$ \\
\hline Fibre $(\mathrm{g})$ & -0.09 & 0.04 & -0.07 & $-0 \cdot 18$ \\
\hline $\mathrm{Na}(\mathrm{mg})$ & 0.33 & $0 \cdot 18$ & $0.51^{\star *}$ & $0 \cdot 36^{*}$ \\
\hline $\mathrm{K}(\mathrm{mg})$ & 0.29 & $0 \cdot 70^{\star \star}$ & $0 \cdot 30$ & $0.54^{\star \star}$ \\
\hline $\mathrm{Ca}(\mathrm{mg})$ & $0 \cdot 41^{*}$ & $0.51^{\star *}$ & $0.45^{\star}$ & $0.43^{\star}$ \\
\hline $\mathrm{Mg}(\mathrm{mg})$ & 0.25 & $0 \cdot 41^{*}$ & $0 \cdot 30$ & $0 \cdot 43^{*}$ \\
\hline$P(\mathrm{mg})$ & $0 \cdot 38^{*}$ & $0 \cdot 4^{*}$ & $0.44^{*}$ & $0.59^{\star *}$ \\
\hline $\mathrm{Fe}(\mathrm{mg})$ & $0 \cdot 10$ & $0.42^{*}$ & 0.32 & 0.33 \\
\hline $\mathrm{Cu}(\mathrm{mg})$ & 0.08 & $-0 \cdot 19$ & $0 \cdot 15$ & $-0 \cdot 14$ \\
\hline Zn (mg) & $0 \cdot 27$ & $0.41^{*}$ & $0.37^{*}$ & $0.45^{*}$ \\
\hline $\mathrm{Cl}(\mathrm{mg})$ & $0.37^{\star}$ & 0.21 & $0.51^{\star *}$ & $0.43^{*}$ \\
\hline $\mathrm{Mn}(\mathrm{mg})$ & $0 \cdot 31$ & $0 \cdot 71^{\star *}$ & $0 \cdot 37^{\star}$ & $0.54^{\star \star}$ \\
\hline $\operatorname{Se}(\mu \mathrm{g})$ & 0.01 & 0.04 & $0 \cdot 15$ & 0.06 \\
\hline lodine $(\mu \mathrm{g})$ & $0 \cdot 48^{\star *}$ & $0.53^{\star \star}$ & $0 \cdot 48^{\star *}$ & $0.42^{*}$ \\
\hline Retinol $(\mu \mathrm{g})$ & $0.44^{*}$ & $0 \cdot 31$ & $0.50^{\star *}$ & 0.06 \\
\hline$\beta$-Carotene equivalents $(\mu \mathrm{g})$ & $0 \cdot 18$ & 0.24 & $0 \cdot 21$ & $0 \cdot 13$ \\
\hline Vitamin D $(\mu \mathrm{g})$ & $0 \cdot 17$ & $0 \cdot 16$ & 0.03 & $0 \cdot 11$ \\
\hline Vitamin E (mg) & 0.03 & $0.39^{*}$ & 0.05 & $0 \cdot 18$ \\
\hline Thiamin (mg) & $0 \cdot 24$ & $0.51^{\star \star}$ & $0 \cdot 20$ & 0.34 \\
\hline Riboflavin (mg) & $0 \cdot 32$ & $0.47^{\star \star}$ & 0.27 & $0 \cdot 47^{\star \star}$ \\
\hline Niacin (mg) & 0.08 & $0 \cdot 20$ & 0.07 & $0 \cdot 15$ \\
\hline Vitamin $B_{6}(\mathrm{mg})$ & $0 \cdot 38^{*}$ & $0.50^{\star *}$ & $0 \cdot 30$ & $0.48^{\star *}$ \\
\hline Vitamin $B_{12}(\mu \mathrm{g})$ & $0 \cdot 46^{\star}$ & $0.51^{\star \star}$ & $0 \cdot 30$ & $0 \cdot 29$ \\
\hline Folic acid $(\mu \mathrm{g})$ & 0.26 & $0 \cdot 42^{*}$ & 0.23 & $0.46^{\star *}$ \\
\hline Pantothenic acid (mg) & $0 \cdot 21$ & $0 \cdot 34$ & 0.24 & $0 \cdot 45^{\star}$ \\
\hline Biotin $(\mu \mathrm{g})$ & $0 \cdot 36^{\star}$ & 0.34 & $0.37^{*}$ & $0.44^{*}$ \\
\hline Vitamin C (mg) & $0 \cdot 40^{\star}$ & $0.69^{\star *}$ & $0.52^{\star \star}$ & $0.52^{\star \star}$ \\
\hline
\end{tabular}

${ }^{*}$ Correlation is significant at the 0.05 level; ${ }^{* *}$ correlation is significant at the 0.01 level.

tFor log-transformed nutrient intakes.

higher estimates than the FD. This corresponds well with findings from other validation studies conducted in pregnant women using similar dietary instruments (FFQ $v$. FD $)^{(17-19)}$. Intakes of nutrients were also found to be higher when determined by FFQ compared with other dietary assessment methods such as $24 \mathrm{~h}$ recall ${ }^{(28)}$. Higher reporting using the FFQ may reflect over-reporting of the frequency of consumption of foods, larger portion size estimation by the FFQ or under-reporting of consumption by the FD or $24 \mathrm{~h}$ recall.

The correlations observed between the SCG-FFQ and FD were comparable with previous studies in pregnancy for our obese participants, and greater than in previous studies for our lean participants ${ }^{(17-19,28)}$, particularly for intakes of total energy, macronutrients and essential nutrients during pregnancy. For example, correlations of measured total energy intake in our obese group were $r_{\mathrm{P}}=0 \cdot 284 / r_{\mathrm{S}}=0.334$ and in our lean group were $r_{\mathrm{P}}=0 \cdot 462 / r_{\mathrm{S}}=0.510$; compared with previous reports of $r_{\mathrm{S}}=0 \cdot 27^{(17)}, r_{\mathrm{P}}=0 \cdot 26^{(28)}, r_{\mathrm{P}}=0 \cdot 24^{(18)}$ and $r_{\mathrm{S}}=0 \cdot 28^{(19)}$ in pregnant women (where $r_{\mathrm{P}}$ is Pearson correlation, $r_{\mathrm{S}}$ is Spearman correlation). The reasons for closer correlation in our lean women are unknown, but may reflect that this was a group of lean women who had consented to participate in a study of obesity in pregnancy, and so were well motivated and interested in health behaviour and weight in pregnancy. A further reason is that we adjusted for total energy intake in order to minimize the effect of measurement errors such as misreporting of energy intake, which would also affect the absolute intakes of nutrients ${ }^{(23)}$. This method of analysis resulted in improved correlation between the two methods. Significant agreement was also seen for important nutrients such as $\mathrm{Ca}, \mathrm{Fe}$, vitamins $\mathrm{E}, \mathrm{B}_{6}, \mathrm{~B}_{12}$ and $\mathrm{C}$, and also for SFA, dietary cholesterol and sugars, in both our obese and lean groups, as observed by others ${ }^{(19)}$. However, correlations for energy-adjusted dietary fibre were poorer in the obese group $\left(r_{\mathrm{P}}=0 \cdot 044 / r_{\mathrm{S}}=-0 \cdot 183\right)$ than the lean group $\left(r_{\mathrm{P}}=0 \cdot 377 / r_{\mathrm{S}}=-0 \cdot 294\right)$, as well as other studies $\left(r_{\mathrm{P}}=0 \cdot 36-0 \cdot 47^{(28)}\right.$ and $\left.r_{\mathrm{P}}=0 \cdot 56^{(18)}\right)$. This suggests 
Table 5 Pearson $\left(r_{\mathrm{P}}\right)$ and Spearman $\left(r_{\mathrm{S}}\right)$ correlation coefficients between the FFQ and $4 \mathrm{~d}$ food diary in the lean group ( $n$ 32): pregnant women between 12 and 29 weeks' gestation, Edinburgh, UK

\begin{tabular}{|c|c|c|c|c|}
\hline \multirow[b]{2}{*}{ Nutrient } & \multicolumn{2}{|c|}{ Pearsont } & \multicolumn{2}{|c|}{ Spearman } \\
\hline & $r_{\mathrm{P}}^{\text {measured }}$ & $r_{\mathrm{P}}^{\text {energy-adjusted }}$ & $r_{\mathrm{S}}^{\text {measured }}$ & $r_{\mathrm{S}}^{\text {energy-adjustec }}$ \\
\hline Energy (kJ) & $0.46^{\star \star}$ & - & $0.51^{\star \star}$ & - \\
\hline Protein $(\mathrm{g})$ & $0.49^{\star \star}$ & $0 \cdot 11$ & $0 \cdot 62^{\star \star \star}$ & $0 \cdot 20$ \\
\hline Fat (g) & 0.30 & 0.04 & $0 \cdot 44^{*}$ & -0.02 \\
\hline Carbohydrate $(\mathrm{g})$ & 0.05 & -0.08 & $0 \cdot 31$ & 0.07 \\
\hline SFA (g) & $0 \cdot 60^{\star * *}$ & $0.53^{\star *}$ & $0 \cdot 66^{\star \star *}$ & $0 \cdot 36^{*}$ \\
\hline MUFA (g) & $0.39^{*}$ & $0 \cdot 31$ & $0 \cdot 38^{*}$ & $0 \cdot 22$ \\
\hline PUFA (g) & $0 \cdot 52^{\star *}$ & $0 \cdot 40^{\star *}$ & $0.58^{\star *}$ & $0 \cdot 41^{*}$ \\
\hline Cholesterol (mg) & $0 \cdot 66^{\star \star \star}$ & $0 \cdot 74^{\star *}$ & $0 \cdot 50^{\star \star}$ & $0 \cdot 46^{\star \star}$ \\
\hline Sugars (g) & 0.08 & $0 \cdot 21$ & $0 \cdot 15$ & $0 \cdot 27$ \\
\hline Starch $(\mathrm{g})$ & $0 \cdot 51^{\star *}$ & $0 \cdot 15$ & $0 \cdot 42^{*}$ & $0 \cdot 20$ \\
\hline Fibre (g) & $0.53^{* *}$ & $0.38^{*}$ & $0 \cdot 40^{*}$ & 0.29 \\
\hline $\mathrm{Na}(\mathrm{mg})$ & $0 \cdot 397^{\star}$ & $0 \cdot 24$ & $0 \cdot 38^{*}$ & 0.22 \\
\hline $\mathrm{K}(\mathrm{mg})$ & $0 \cdot 38^{*}$ & $0.52^{\star \star}$ & $0.45^{\star *}$ & $0.49^{\star \star}$ \\
\hline $\mathrm{Ca}(\mathrm{mg})$ & $0 \cdot 59^{\star \star \star}$ & $0 \cdot 37^{\star}$ & $0 \cdot 60^{\star \star \star}$ & $0 \cdot 32$ \\
\hline $\mathrm{Mg}(\mathrm{mg})$ & $0.39^{*}$ & $0 \cdot 18$ & 0.34 & $0 \cdot 29$ \\
\hline$P(\mathrm{mg})$ & $0.56^{\star \star}$ & $0.45^{\star *}$ & $0.52^{* *}$ & $0 \cdot 34$ \\
\hline $\mathrm{Fe}(\mathrm{mg})$ & $0.59^{\star \star \star}$ & 0.23 & $0 \cdot 47^{\star \star}$ & 0.26 \\
\hline $\mathrm{Cu}(\mathrm{mg})$ & 0.04 & -0.25 & $0 \cdot 13$ & -0.08 \\
\hline $\mathrm{Zn}(\mathrm{mg})$ & $0 \cdot 66^{\star \star \star}$ & $0 \cdot 40^{\star}$ & $0 \cdot 64^{\star \star \star}$ & $0.43^{*}$ \\
\hline $\mathrm{Cl}(\mathrm{mg})$ & $0 \cdot 45^{\star}$ & $0 \cdot 27$ & $0 \cdot 39^{\star}$ & $0 \cdot 20$ \\
\hline $\mathrm{Mn}(\mathrm{mg})$ & $0 \cdot 63^{\star \star \star}$ & $0.68^{\star *}$ & $0 \cdot 61^{\star \star \star}$ & $0.57^{\star \star}$ \\
\hline Se $(\mu \mathrm{g})$ & 0.22 & 0.29 & 0.25 & 0.28 \\
\hline lodine $(\mu \mathrm{g})$ & $0 \cdot 52^{\star \star}$ & $0.43^{*}$ & $0.52^{* *}$ & $0 \cdot 38^{\star}$ \\
\hline Retinol $(\mu \mathrm{g})$ & 0.25 & 0.32 & $0 \cdot 44^{*}$ & $0.43^{*}$ \\
\hline$\beta$-Carotene equivalents $(\mu \mathrm{g})$ & $0.43^{*}$ & $0 \cdot 40^{*}$ & 0.33 & $0 \cdot 27$ \\
\hline Vitamin D $(\mu \mathrm{g})$ & $0 \cdot 16$ & $0 \cdot 25$ & 0.22 & $0 \cdot 21$ \\
\hline Vitamin $\mathrm{E}(\mathrm{mg})$ & $0 \cdot 52^{\star *}$ & $0 \cdot 31$ & $0 \cdot 48^{\star \star}$ & $0 \cdot 28$ \\
\hline Thiamin (mg) & $0 \cdot 46^{\star *}$ & 0.34 & $0 \cdot 38^{*}$ & $0 \cdot 22$ \\
\hline Riboflavin (mg) & $0.52^{\star \star}$ & $0 \cdot 37^{\star}$ & $0 \cdot 37^{*}$ & 0.33 \\
\hline Niacin (mg) & $0.53^{\star *}$ & $0.56^{\star \star}$ & $0 \cdot 51^{* *}$ & $0 \cdot 25$ \\
\hline Vitamin $B_{6}(\mathrm{mg})$ & $0.49^{\star *}$ & $0.42^{*}$ & $0 \cdot 50^{\star \star}$ & $0 \cdot 45^{\star \star}$ \\
\hline Vitamin $B_{12}(\mu \mathrm{g})$ & $0 \cdot 39^{*}$ & $0 \cdot 34$ & $0 \cdot 24$ & $0 \cdot 14$ \\
\hline Folic acid $(\mu \mathrm{g})$ & $0.39^{*}$ & 0.34 & $0 \cdot 36^{*}$ & $0 \cdot 23$ \\
\hline Pantothenic acid (mg) & $0 \cdot 50^{\star *}$ & $0.42^{\star}$ & $0.43^{*}$ & $0 \cdot 17$ \\
\hline Biotin $(\mu \mathrm{g})$ & $0 \cdot 48^{\star \star}$ & $0.43^{*}$ & $0 \cdot 44^{*}$ & $0 \cdot 32$ \\
\hline Vitamin C (mg) & 0.33 & $0 \cdot 45^{\star}$ & $0 \cdot 37^{*}$ & $0.37^{*}$ \\
\hline
\end{tabular}

${ }^{*}$ Correlation is significant at the 0.05 level; ${ }^{* *}$ correlation is significant at the 0.01 level; ${ }^{* \star *}$ correlation is significant at the 0.001 level.

+For log-transformed nutrient intakes.

possible over-reporting of portion size/frequency of dietary fibre intake in the FFQ as opposed to underreporting in the FD; it has been reported that fruits and vegetables are perceived as healthy and are more likely to be over-reported in $\mathrm{FFQ}^{(29)}$.

The ability of the SCG-FFQ to rank individuals by intake was evaluated by the percentage of women who were classified into the same or opposite tertiles of (energy-adjusted) intake by both methods. Overall, in the present study, about half of the participants were correctly classified and about $10 \%$ were grossly misclassified into the opposite tertile. Comparable results were reported by Robinson et al. ${ }^{(19)}$ who cross-classified using quintiles and obtained an average of $35 \%$ consistent classification and $6 \%$ gross misclassification. Others have reported higher percentages (between 49 and 94\%) of consistent classification using the weighed food record $^{(17)}$ and $24 \mathrm{~h}$ diet recall ${ }^{(28)}$ as reference method. These techniques may have advantages such as better food portion estimation (from weighing) and more complete information (from multiple-pass dietary interviews) compared with an unweighed FD.

The $\kappa_{\mathrm{w}}$ statistic tests ranking agreement between the FFQ and the reference method, taking partial agreement into consideration ${ }^{(22)}$. The $\kappa_{\mathrm{w}}$ value for total energy was lower in obese women as compared with lean. The $\kappa_{\mathrm{w}}$ values were found to show poor agreement for fibre in the obese group, and slight agreement for $\mathrm{Cu}$, Se and vitamin $\mathrm{B}_{12}$ in the lean group. The highest $\boldsymbol{\kappa}_{\mathrm{w}}$ values were found for SFA in obese women and $\mathrm{Ca}$ and $\mathrm{Zn}$ in lean women, whereas the lowest $\kappa_{\mathrm{w}}$ values were observed for dietary fibre in obese and $\mathrm{Cu}$ in lean women, in agreement with the results obtained from cross-classification and correlation methods. This is consistent with overreporting of foods perceived to be 'more healthy' using the $\mathrm{FFQ}^{(29)}$.

The use of a second group of lean pregnant women allowed comparison of our results with similar populations reported in the literature, demonstrating that the obese and lean groups did in fact behave differently in 
Table 6 Percentages of women classified into the same and opposite tertiles of intake (according to the FFQ and the $4 \mathrm{~d}$ food diary) and weighted kappa values $\left(\kappa_{\mathrm{w}}\right)$ : pregnant women between 12 and 29 weeks' gestation, Edinburgh, UK

\begin{tabular}{|c|c|c|c|c|c|c|c|c|}
\hline \multirow[b]{3}{*}{ Nutrient } & \multicolumn{4}{|c|}{ Obese $(n 31)$} & \multicolumn{4}{|c|}{ Lean ( $n$ 32) } \\
\hline & \multicolumn{2}{|c|}{$\%$ classified in } & \multirow[b]{2}{*}{$\kappa_{\mathrm{w}}$} & \multirow[b]{2}{*}{$P$ value } & \multicolumn{2}{|c|}{$\%$ classified in } & \multirow[b]{2}{*}{$\kappa_{\mathrm{w}}$} & \multirow[b]{2}{*}{$P$ value } \\
\hline & Same tertile & Opposite tertiles & & & Same tertile & Opposite tertiles & & \\
\hline Energy (kJ) & 45 & 10 & 0.468 & 0.044 & 50 & 12 & 0.560 & 0.013 \\
\hline Protein $(g)$ & 42 & 10 & 0.471 & 0.043 & 53 & 3 & 0.743 & $<0.001$ \\
\hline Fat $(g)$ & 61 & 6 & 0.687 & 0.001 & 50 & 12 & 0.559 & 0.013 \\
\hline Carbohydrate (g) & 52 & 19 & 0.373 & $0 \cdot 103$ & 44 & 16 & 0.321 & $0 \cdot 143$ \\
\hline SFA (g) & 64 & 3 & 0.789 & $<0.001$ & 53 & 3 & 0.744 & $<0.001$ \\
\hline MUFA (g) & 55 & 3 & $0 \cdot 721$ & $<0.001$ & 38 & 16 & $0 \cdot 361$ & $0 \cdot 109$ \\
\hline PUFA (g) & 48 & 6 & 0.552 & 0.016 & 50 & 9 & 0.634 & 0.003 \\
\hline Cholesterol (mg) & 45 & 13 & 0.483 & 0.038 & 50 & 6 & 0.674 & 0.001 \\
\hline Sugars (g) & 48 & 10 & 0.520 & 0.024 & 31 & 16 & 0.282 & $0 \cdot 180$ \\
\hline Starch $(\mathrm{g})$ & 58 & 13 & 0.527 & 0.022 & 47 & 12 & 0.498 & 0.030 \\
\hline Fibre $(\mathrm{g})$ & 26 & 13 & -0.039 & 0.541 & 50 & 12 & 0.548 & 0.015 \\
\hline $\mathrm{Na}(\mathrm{mg})$ & 48 & 10 & 0.560 & 0.014 & 41 & 6 & 0.589 & 0.008 \\
\hline $\mathrm{K}(\mathrm{mg})$ & 58 & 16 & 0.498 & 0.032 & 47 & 9 & 0.550 & 0.015 \\
\hline $\mathrm{Ca}(\mathrm{mg})$ & 45 & 13 & 0.508 & 0.028 & 56 & 0 & 0.775 & $<0.001$ \\
\hline $\mathrm{Mg}(\mathrm{mg})$ & 42 & 13 & 0.409 & 0.078 & 53 & 9 & 0.578 & 0.009 \\
\hline$P(\mathrm{mg})$ & 55 & 13 & 0.586 & 0.009 & 47 & 3 & 0.675 & 0.001 \\
\hline $\mathrm{Fe}(\mathrm{mg})$ & 32 & 13 & 0.269 & $0 \cdot 198$ & 50 & 6 & 0.626 & 0.004 \\
\hline $\mathrm{Cu}(\mathrm{mg})$ & 45 & 19 & $0 \cdot 201$ & 0.272 & 34 & 16 & 0.003 & 0.496 \\
\hline $\mathrm{Zn}(\mathrm{mg})$ & 45 & 13 & 0.465 & 0.046 & 62 & 3 & 0.765 & $<0.001$ \\
\hline $\mathrm{Cl}(\mathrm{mg})$ & 55 & 6 & $0 \cdot 713$ & $<0.001$ & 41 & 9 & 0.471 & 0.041 \\
\hline $\mathrm{Mn}(\mathrm{mg})$ & 61 & 13 & 0.539 & $0 \cdot 019$ & 56 & 22 & 0.645 & 0.003 \\
\hline $\operatorname{Se}(\mu \mathrm{g})$ & 55 & 13 & 0.481 & 0.039 & 38 & 22 & $0 \cdot 163$ & 0.311 \\
\hline lodine $(\mu \mathrm{g})$ & 52 & 6 & 0.636 & 0.004 & 53 & 9 & 0.649 & 0.002 \\
\hline Retinol $(\mu \mathrm{g})$ & 61 & 10 & 0.673 & 0.002 & 56 & 3 & 0.660 & 0.002 \\
\hline$\beta$-Carotene equivalents $(\mu \mathrm{g})$ & 35 & 13 & $0 \cdot 306$ & $0 \cdot 161$ & 44 & 12 & 0.467 & 0.043 \\
\hline Vitamin D $(\mu \mathrm{g})$ & 39 & 16 & 0.093 & 0.395 & 47 & 16 & 0.426 & 0.064 \\
\hline Vitamin E (mg) & 35 & 16 & 0.247 & 0.221 & 41 & 12 & 0.435 & 0.059 \\
\hline Thiamin (mg) & 48 & 22 & 0.226 & 0.244 & 44 & 9 & 0.500 & 0.029 \\
\hline Riboflavin (mg) & 48 & 16 & 0.409 & 0.078 & 41 & 9 & 0.421 & 0.067 \\
\hline Niacin (mg) & 48 & 39 & $0 \cdot 250$ & $0 \cdot 217$ & 53 & 6 & 0.656 & 0.002 \\
\hline Vitamin $B_{6}(\mathrm{mg})$ & 58 & 13 & 0.566 & 0.013 & 56 & 6 & $0 \cdot 708$ & $<0.001$ \\
\hline Vitamin $B_{12}(\mu \mathrm{g})$ & 35 & 10 & 0.408 & 0.078 & 38 & 22 & 0.163 & 0.311 \\
\hline Folic acid $(\mu \mathrm{g})$ & 45 & 16 & $0 \cdot 215$ & 0.255 & 47 & 9 & 0.586 & 0.008 \\
\hline Pantothenic acid (mg) & 48 & 16 & 0.472 & 0.043 & 41 & 6 & 0.487 & 0.034 \\
\hline Biotin $(\mu \mathrm{g})$ & 52 & 10 & 0.543 & 0.018 & 50 & 9 & 0.568 & 0.011 \\
\hline Vitamin C (mg) & 58 & 13 & 0.595 & 0.008 & 50 & 9 & 0.566 & 0.011 \\
\hline
\end{tabular}

our study. The main limitation was that both the FFQ and FD were self-reported and therefore could be subject to similar measurement error and reporting bias. The use of an FD may also not represent habitual intake, as it may influence changes in eating behaviour through the act of recording ${ }^{(6)}$. In addition, responses to the FFQ and FD may differ in early pregnancy from later pregnancy due to nausea, sensitivity to food smells, vomiting, bloating and heartburn. It has been suggested that over-reporting could be due to either over-reporting of food portions and frequency in the FFQ, or under-reporting in the reference method ${ }^{(19)}$. Under-reporting of food intake has been found to be particularly prevalent in obese ${ }^{(15,30)}$ and pregnant ${ }^{(31,32)}$ women and occurs across dietary measurement instruments ${ }^{(33)}$. Since under-reporting most commonly involves recording of implausibly low food intake (evidenced by low total energy intake), this also affects estimates of absolute nutrient intakes. By adjusting for reported total energy intake, the proportion of the diet composed of each nutrient is represented. This reduces the possibility of measurement error caused by misreporting of total energy intake, making nutrient intakes independent of total energy ${ }^{(15)}$. As there is no 'gold standard' in dietary measurement, comparisons between measurement methods can only be relative, rather than assessing the accuracy of each instrument.

It has been demonstrated that the method of administration of an FFQ may affect the validity of results. Correlations between FFQ and a reference method have been reported to be higher for interviewer-administered than self-administered FFQ for certain nutrients ${ }^{(16)}$. Interviewer-administered methods may be advantageous as they allow immediate checking for any missing or incomprehensible response by the interviewer. However, the cost, time and resources involved must be considered. In addition, the presence of an interviewer may increase the likelihood of social desirability bias in the participants' responses. Discussing dietary patterns in such obese pregnant women is clearly a sensitive issue as demonstrated by our high attrition rate in the obese group. 


\section{Conclusions}

The validity of the SCG-FFQ was found to be lower in obese than in lean pregnant Scottish women, as demonstrated by poorer agreement between the FFQ and FD. The SCG-FFQ was, however, able to rank individuals by levels of nutrient intakes, and was better for some nutrients such as SFA, dietary cholesterol, sugars and vitamin $\mathrm{C}$, but poorer for other such as dietary fibre, in severely obese pregnant women. Overall, the SCG-FFQ appears to be a suitable tool for evaluating food intake during pregnancy in severely obese pregnant women.

\section{Acknowledgements}

Sources of funding: The study was funded by Tommy's The Baby Charity and the Malaysian Ministry of Higher Education. Conflict of interest: The authors declare that they have no conflicts of interest. Authors' contributions: R.M.R., J.E.N. and B.R.W. designed the research. N.A.M.-S. conducted the research, decoded the SCG-FFQ data, analysed the FD data and carried out the statistical analyses. J.L.B. provided statistical support. N.A.M.-S. had primary responsibility for writing the manuscript. All authors read and approved the final manuscript. Acknowledgements: The authors acknowledge the support of the British Heart Foundation Centre of Research Excellence.

\section{References}

1. Butte NF, Wong WW, Treuth MS et al. (2004) Energy requirements during pregnancy based on total energy expenditure and energy deposition. Am J Clin Nutr 79, 1078-1087.

2. WHO Media Centre (2011) Obesity and overweight fact sheet. http://www.who.int/mediacentre/factsheets/fs311/ en/index.html (accessed August 2011).

3. Corbett J, Given L, Gray L et al. (2009) The Scottish Health Survey 2008. Volume I: Main Report. Edinburgh: The Scottish Government.

4. Davies GA, Maxwell C, McLeod L et al. (2010) Obesity in pregnancy. J Obstet Gynaecol Can 32, 165-173.

5. Wakai K (2009) A review of food frequency questionnaires developed and validated in Japan. J Epidemiol 19, 1-11.

6. Cade J, Thompson R, Burley V et al. (2002) Development, validation and utilisation of food-frequency questionnaires - a review. Public Health Nutr 5, 567-587.

7. Hure A, Young A, Smith R et al. (2009) Diet and pregnancy status in Australian women. Public Health Nutr 12, 853-861.

8. Mouratidou T, Ford F, Prountzou F et al. (2006) Dietary assessment of a population of pregnant women in Sheffield, UK. BrJ Nutr 96, 929-935.

9. Martindale S, McNeill G, Devereux G et al. (2005) Antioxidant intake in pregnancy in relation to wheeze and eczema in the first two years of life. Am J Respir Crit Care Med 171, 121-128.

10. Moore VM, Davies MJ, Willson KJ et al. (2004) Dietary composition of pregnant women is related to size of the baby at birth. J Nutr 134, 1820-1826.

11. Mathews M, Yudkin P \& Neil A (1999) Influence of maternal nutrition on outcome of pregnancy: prospective cohort study. BMJ 319, 339-343.
12. Rogers I \& Emmett P (1998) Diet during pregnancy in a population of pregnant women in south west England. ALSPAC study team. Avon longitudinal study of pregnancy and childhood. Eur J Clin Nutr 52, 246-250.

13. Godfrey K, Robinson S, Barker DJ et al. (1996) Maternal nutrition in early and late pregnancy in relation to placental and fetal growth. BMJ 312, 410-414.

14. Kipnis V, Midthune D, Freedman L et al. (2002) Bias in dietary-report instruments and its implications for nutritional epidemiology. Public Health Nutr 5, 915-923.

15. Poslusna K, Ruprich J, de Vries JH et al. (2009) Misreporting of energy and micronutrient intake estimated by food records and 24 hour recalls, control and adjustment methods in practice. Br J Nutr 101, Suppl. 2, S73-S85.

16. Cade JE, Burley VJ, Warm DL et al. (2004) Food-frequency questionnaires: a review of their design, validation and utilisation. Nutr Res Rev 17, 5-22.

17. Brantsaeter AL, Haugen M, Alexander J et al. (2008) Validity of a new food frequency questionnaire for pregnant women in the Norwegian mother and child cohort study (MOBA). Matern Child Nutr 4, 28-43.

18. Erkkola M, Karppinen M, Javanainen J et al. (2001) Validity and reproducibility of a food frequency questionnaire for pregnant Finnish women. Am J Epidemiol 154, 466-476.

19. Robinson S, Godfrey K, Osmond C et al. (1996) Evaluation of a food frequency questionnaire used to assess nutrient intakes in pregnant women. Eur J Clin Nutr 50, 302-308.

20. Masson LF, McNeill G, Tomany JO et al. (2003) Statistical approaches for assessing the relative validity of a foodfrequency questionnaire: use of correlation coefficients and the kappa statistic. Public Health Nutr 6, 313-321.

21. Anderson LF, Tomten H, Haggarty P et al. (2003) Validation of energy intake estimated from a food frequency questionnaire: a doubly labelled water study. Eur J Clin Nutr 57, 279-284.

22. Willett W \& Stampfer MJ (1986) Total energy intake: implications for epidemiologic analyses. Am J Epidemiol 124, 17-27.

23. Norman GR \& Streiner DL (2008) Biostatistics: The Bare Eessentials. New York: McGraw-Hill.

24. McLoone P \& Boddy FA (1994) Deprivation and mortality in Scotland 1981 and 1991. BMJ 309, 1465-1470.

25. Brunner E, Stallone D, Juneja M et al. (2001) Dietary assessment in WHITEHALL II: comparison of $7 \mathrm{~d}$ diet diary and food-frequency questionnaire and validity against biomarkers. Br J Nutr 86, 405-414.

26. Landis JR \& Koch GG (1977) An application of hierarchical kappa-type statistics in the assessment of majority agreement among multiple observers. Biometrics 33, 363-374.

27. Sempos CT, Briefel RR, Johnson C et al. (1992) Process and rationale for selecting dietary methods for NHANES III. Vital Health Stat 4 issue 27, 85-90.

28. Mouratidou T, Ford F \& Fraser R (2006) Validation of a food-frequency questionnaire for use in pregnancy. Public Health Nutr 9, 515-522.

29. Johansson L, Solvoll K, Bjørneboe GE et al. (1998) Underand overreporting of energy intake related to weight status and lifestyle in a nationwide sample. Am J Clin Nutr 68, 266-274.

30. Ferrari P, Slimani N, Ciampi A et al. (2002) Evaluation of under- and overreporting of energy intake in the 24-hour diet recalls in the European Prospective Investigation into Cancer and Nutrition (EPIC). Public Health Nutr 5, 1329-1345.

31. Forsum E \& Löf M (2007) Energy metabolism during human pregnancy. Annu Rev Nutr 27, 277-292.

32. Derbyshire E, Davies J, Costarelli V et al. (2006) Prepregnancy body mass index and dietary intake in the first trimester of pregnancy. J Hum Nutr Diet 19, 267-273.

33. Black AE \& Cole TJ (2001) Biased over- or under-reporting is characteristic of individuals whether over time or by different assessment methods. J Am Diet Assoc 101, 70-80. 